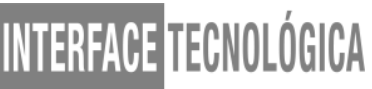

\section{UM ESTUDO SOBRE A IMPORTÂNCIA DO APPCC - ANÁLISE DE PERIGOS E PONTOS CRÍTICOS DE CONTROLE - NA INDÚSTRIA DE ALIMENTOS}

\author{
A STUDY ON THE IMPORTANCE OF HACCP - ANALYSIS OF HAZARDS AND \\ CRITICAL CONTROL POINTS - IN THE FOOD INDUSTRY
}

Sara da Silva Quintino - sarahquintino2013@hotmail.com
Daniela Rodolpho - danirodolpho@yahoo.com.br

Faculdade de Tecnologia de Taquaritinga (FATEC) - SP - Brasil

DOI: $10.31510 /$ infa.v15i2.452

\begin{abstract}
RESUMO
A produção de alimentos seguros se tornou uma necessidade nas empresas devido à concorrência de mercado e exigência do consumidor. Garantir a qualidade de um alimento se refere ao atendimento das especificações e características do produto, enquanto a garantia de produção de um alimento seguro se refere à análise de todo o processo, prevenindo as causas de contaminação física, química ou microbiológica, que podem afetar a saúde do consumidor. Assim, o presente estudo consiste em uma análise bibliográfica e documental que mostra os princípios e a importância do sistema APPCC - Análise de Perigos e Pontos Críticos de Controle - na indústria de alimentos. O APPCC é uma metodologia que direciona esse estudo, para detecção dos pontos críticos de controle na fabricação de alimentos e possibilita o monitoramento, eliminação ou diminuição dos riscos/perigo a um nível aceitável no processo produtivo. As boas práticas de fabricação e os procedimentos operacionais padronizados são pré-requisitos para a implantação do APPCC, uma vez que visam à qualidade do produto desde a entrada da matéria-prima, processamento, chegando até o consumidor. Portanto, a implantação do APPCC na indústria alimentícia propicia uma série de benefícios, tais como aumento da qualidade do produto, maior produtividade com menor índice de retrabalho e redução do número de reclamações de consumidores, além de proporcionar um diferencial à indústria, quanto à certificação de qualidade, valorizando seu produto e proporcionando sua aceitação no mercado.
\end{abstract}

Palavras-chave: Práticas. Controle. Segurança.

\begin{abstract}
Safe food production has become a necessity in business because of market competition and consumer demand. Ensure food quality refers to the fulfillment of the product specifications and characteristics, while the production assurance of a safe food refers to analysis of whole process, preventing the causes of physical, chemical or microbiological contamination that can affect the consumer health. Thus, this study consists of a bibliographical and documentary analysis that shows the principles and importance of the HACCP system - Hazard Analysis and Critical Control Points - in the food industry. HACCP is a methodology that directs this
\end{abstract}


study to detect the critical points in the food manufacturing control and allows the monitoring, elimination or reduction of risks/hazards to an acceptable level in the production process. The good manufacturing practices and standard operating procedures are prerequisites for the HACCP implementation, since they aim at product quality from the input of the raw material, processing, to the consumer. Therefore, HACCP implementation in the food industry provides a series of advantages, such as increased product quality, higher productivity with lower rework rate and reduction of consumer complaints, as well as providing a differential to the industry, regarding certification quality, valuing its product and providing its acceptance in the market.

Keywords: Practices. Control. Safety.

\section{INTRODUÇÃO}

Produzir um alimento seguro inclui o atendimento a legislações e adoção de metodologias de qualidade para análise de todo o processo de fabricação, prevenindo as causas de contaminação física, química ou microbiológica, que podem afetar a saúde do consumidor.

A RDC 275, de 21 de outubro de 2002, estabelece os Procedimentos Operacionais Padronizados (POP's), que são instruções sequenciais escritas de forma objetiva para a realização de operações rotineiras e específicas na produção, armazenamento e transporte de alimentos (BRASIL, 2002).

As Boas Práticas de Fabricação (BPF) são normas de procedimentos para atingir um determinado padrão de identidade e qualidade de um produto ou serviço na área de alimentos, avaliada através de inspeções e aplicada a todos os estabelecimentos produtores e/ou prestadores de serviços na área de alimentos (BRASIL, 1993).

O APPCC é uma abordagem científica e sistemática para o controle de processo, elaborado para prevenir a ocorrência de problemas, assegurando que controles sejam aplicados em determinadas etapas no sistema de produção de alimentos, onde possam ocorrer perigos ou situações críticas (BRASIL, 1998).

Da sigla APPCC, o “AP” significa Análise de Perigos, e torna-se a parte principal do sistema, assim como a determinação dos PCCs que são os Pontos Críticos de Controle (DIAS; BARBOSA; COSTA, 2010 apud VERGARA, 2015, p. 99).

A realização do presente estudo consiste em uma análise bibliográfica e documental, com o objetivo de apresentar os princípios para implantação do APPCC e sua importância na indústria de alimentos. O artigo é estruturado em seis seções para a apresentação do estudo, 
cuja relevância é mostrar o APPCC como um diferencial na indústria alimentícia, garantindo a qualidade e segurança do produto, proporcionando sua aceitação no mercado e atendendo às exigências internacionais.

\section{A IMPORTÂNCIA DO APPCC NA INDÚSTRIA DE ALIMENTOS}

O APPCC tem grande importância na indústria de alimentos, pois atua de forma preventiva garantindo um produto livre de contaminantes físicos, químicos ou biológicos. A análise realizada nas etapas durante a fabricação permite um controle antes da transformação final do produto, permitindo a adoção de medidas corretivas e preventivas para eliminação dos perigos de contaminação. Um controle rigoroso no processo de fabricação do alimento proporciona maior qualidade, segurança, redução de perdas e retrabalho no processo produtivo.

O termo alimento seguro é um conceito de grande importância para a saúde pública e para o comércio internacional (BARENDSZ, 1998 apud FIGUEIREDO; COSTA NETO, 2001, p. 101).

A qualidade para a indústria de alimentos tem como fator determinante a segurança do produto, proporcionando uma vantagem competitiva entre as empresas. Os consumidores estão cada vez mais exigentes ao adquirir um determinado produto, portanto, as empresas que não estiverem preocupadas com esta busca pela qualidade poderão ficar à margem do mercado consumidor (FIGUEIREDO; COSTA NETO, 2001 p. 101).

O comprometimento da alta direção, o envolvimento e treinamento de todos colaboradores da empresa são fatores de extrema importância para que o sistema APPCC tenha sucesso em sua implantação, garantindo a qualidade e segurança do alimento.

Alguns segmentos possuem obrigatoriedade legal para implantação do APPCC devido às possíveis contaminações, mas é interessante ressaltar que qualquer indústria de alimentos pode implantar o sistema, pois uma empresa certificada melhora sua imagem no mercado nacional e internacional aumentando a confiança dos consumidores em relação à qualidade e segurança do produto adquirido. Pode-se afirmar que qualidade significa sobrevivência no mercado (GERMANO; GERMANO, 2011 apud VERGARA, 2015, p. 99). 


\section{AS bOAS PRÁticas de FABRICAÇÃo COMO PRÉ-REQUISITO PARA O APPCC}

No processo de fabricação de alimentos é essencial a aplicação de conceitos de boas práticas de higiene e manipulação para a garantia da qualidade do produto. O APPCC deve ser acompanhado do programa de pré-requisitos que fornecerá as condições operacionais e ambientais básicas para a produção de alimentos seguros (BERTHIER, 2007 apud COLLETO, 2012, p. 18).

Para a obtenção de um alimento com qualidade e segurança, cuidados devem ser tomados em relação à higiene e limpeza das instalações, dos equipamentos de produção, de utensílios utilizados na fabricação, dos manipuladores e todos envolvidos no processo. As BPF são medidas higiênicas necessárias para evitar contaminação ao alimento (como utilização de toucas cobrindo todo o cabelo, higienização correta das mãos, unhas cortadas e limpas, utilização de uniforme limpo e exclusivamente nas dependências da empresa, ausência de adornos, perfume), entre outras medidas de prevenção.

A RDC 275, de 21 de outubro de 2002, estabelece os POP's para a garantia de condições higiênico-sanitárias que complementam as BPF para a produção de alimentos (BRASIL, 2002).

O estabelecimento e cumprimento dos POP's como de controle de pragas, potabilidade da água, rastreabilidade, recolhimento de produtos, produtos não conformes, limpeza, higiene de colaboradores, qualificação de fornecedores, contribuem para a qualidade do produto, pois atuam em todo o processo produtivo.

Segundo a Portaria 368, de 4 de setembro de 1997, as BPF são os procedimentos necessários para a obtenção de alimentos inócuos e saudáveis (BRASIL, 1997). Toda matériaprima a ser utilizada no processo de fabricação deve ser de boa procedência, qualificada e analisada antes de sua utilização, incluindo as bebidas, aditivos, embalagens, utensílios e materiais em contato com alimentos.

A Agência Nacional de Vigilância Sanitária (ANVISA) dispõe de normas gerais e específicas no processamento de alimentos para garantir a qualidade sanitária e a conformidade dos alimentos com os regulamentos técnicos.

As Portarias 1428/1993 e 326/1997 determinam requisitos gerais sobre as condições higiênico-sanitárias e de BPF para estabelecimentos produtores/industrializadores de alimentos e prestação de serviços na área alimentícia (BRASIL, 1993 e 1997). 


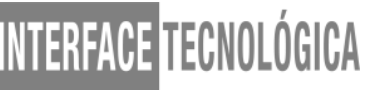

As BPF e o estabelecimento dos POP's são pré-requisitos para o APPCC, pois servem de estrutura para obtenção dos resultados esperados com a adoção do sistema APPCC na indústria alimentícia.

\section{ETAPAS PARA IMPLANTAÇÃO DO APPCC}

Para a implantação do APPCC segue-se uma lógica ordenada de sete princípios básicos, porém antes da aplicação destes princípios existe a necessidade da adoção de algumas etapas preliminares (BRASIL, 1998).

A formação de uma equipe multidisciplinar é a primeira etapa, onde a junção de conhecimentos e experiências contribui no estudo de todo o processo da cadeia produtiva.

Na segunda etapa, o plano APPCC é apresentado com a identificação completa da empresa, organograma claro e com funções definidas.

A terceira etapa consiste em avaliar os pré-requisitos necessários através de uma análise das condições do estabelecimento. Os pré-requisitos envolvem aspectos relacionados à estrutura, instalações, higiene de manipuladores, equipamentos e utensílios abordados pelas BPF e POP's dispostos por legislação.

$\mathrm{Na}$ quarta etapa um programa de capacitação técnica é executado para que todos os envolvidos possam ser treinados continuamente e estar apto no desenvolvimento e manutenção do sistema APPCC.

A aplicação dos sete princípios do sistema APPCC é realizada na quinta etapa.

$\mathrm{Na}$ sexta etapa a documentação do sistema APPCC é encaminhada para aprovação. Produtos de origem animal como leite, pescado e carne são aprovados pelo DIPOA (Departamento de Inspeção de Produtos de Origem Animal), enquanto os demais segmentos de alimentos que não são de origem animal são aprovados pela ANVISA.

A validação é a última etapa, onde o estabelecimento passa por auditoria para verificação do funcionamento do sistema APPCC.

\section{PRINCÍPIOS DO SISTEMA APPCC}

A Portaria 46, do MAPA de 10 de fevereiro de 1998, constitui sete princípios básicos para a implantação do APPCC: Identificação do Perigo, Identificação do Ponto Crítico, 


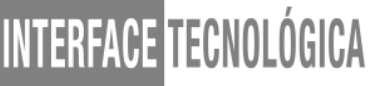

Estabelecimento do limite crítico, Monitorização, Ações corretivas, Procedimentos de verificação e Registros de resultados (BRASIL, 1998).

\subsection{Primeiro Princípio: Identificação do Perigo}

Perigo à segurança de alimento pode ser um agente biológico, químico ou físico, ou condição do alimento, com potencial de causar um efeito adverso à saúde, incluindo os alergênicos. (ABNT NBR ISO 22000:2006).

São causas potenciais de danos inaceitáveis que possam tornar o alimento impróprio para o consumo humano, afetando a saúde, ocasionando perda da qualidade e integridade econômica do produto, como contaminantes biológicos, químicos ou físicos (BRASIL, 1998).

Neste princípio os perigos significativos são identificados e listados em todas as etapas do processo, desde as matérias-primas até o momento do consumo, considerando os fatores de probabilidade de ocorrência e severidade que possam causar danos à saúde. A avaliação qualitativa e quantitativa da presença de perigos, a existência de microrganismos, toxinas e agentes químicos, físicos são essenciais para o estabelecimento de medidas que possam prevenir eliminar ou reduzir a limites aceitáveis qualquer tipo de contaminação ao alimento.

Contaminantes biológicos se caracterizam pela presença de microrganismos patogênicos (que causam dano à saúde). Os contaminantes químicos podem ser denominados como uma formação inaceitável de substâncias químicas em produtos acabados, na linha de produção ou no ambiente e os contaminantes físicos são presença de materiais estranhos ao produto. A contaminação microbiológica pode ser controlada através da higiene durante o manuseio e processamento dos alimentos, enquanto a contaminação química é em geral bastante difícil de ser controlada (BARENDSZ, 1998 apud FIGUEIREDO; COSTA NETO, 2001, p. 102).

A probabilidade de ocorrência de um perigo à saúde é denominada de risco. Os riscos devem ser analisados quanto à sua importância para a saúde pública, considerando a ligação epidemiológica do produto em relação às ETAs - Enfermidades Transmitidas por Alimentos (BRASIL, 1998).

$\mathrm{O}$ perigo ocorre por falhas ou variações significantes no processo, enquanto a severidade é definida como uma consequência gerada por essa falha ou variação que pode ser grave, moderada ou baixa. As informações utilizadas para a condução da identificação dos perigos são devidamente documentadas e os registros mantidos. 


\subsection{Segundo Princípio: Identificação do Ponto Crítico}

Neste princípio é necessária a elaboração do diagrama operacional do produto, para encontrar os pontos de perigo e estabelecer medidas de controle para redução ou eliminação da contaminação. Para a identificação dos pontos críticos a aplicação da árvore decisória consiste em fazer uma série de perguntas sobre cada etapa do de fabricação, tendo como base o diagrama ou fluxograma de processo.

A Portaria 46, do MAPA de 10 de fevereiro de 1998, descreve quatro perguntas utilizadas na aplicação da árvore decisória para a identificação dos PCCs (BRASIL, 1998):

Pergunta 1 - Existem medidas preventivas para o controle dos perigos identificados?

Pergunta 2 - Esta etapa foi especialmente desenvolvida para eliminar ou reduzir a provável ocorrência de um perigo a um nível aceitável?

Pergunta 3 - Poderia o perigo identificado ocorrer em níveis maiores que os aceitáveis, alcançando níveis indesejáveis?

Pergunta 4 - Existe uma etapa subsequente que poderia eliminar o perigo ou reduzir sua ocorrência a níveis aceitáveis?

Os pontos de controle (PC) são pontos controlados em partes do processo ou equipamento para prevenção de um desvio nos pontos críticos de controle (PCC), enquanto o ponto crítico de controle refere-se a uma etapa onde um controle essencial pode ser aplicado para prevenir, eliminar ou reduzir a um limite aceitável um perigo à segurança de alimentos.

O ponto crítico de controle é a última etapa de um processo, capaz de prevenir, eliminar, ou reduzir a níveis aceitáveis um perigo relativo à segurança de alimentos (ABNT NBR ISSO 22000: 2006).

\subsection{Terceiro Princípio: Estabelecimento do Limite Crítico}

O estabelecimento dos limites críticos é determinado para o monitoramento de cada PCC, com o objetivo de assegurar que o nível aceitável do perigo à segurança de alimentos não seja excedido.

O limite crítico é o critério que separa a aceitação da rejeição do produto. Os limites devem ser mensuráveis, pois separaram os produtos aceitáveis dos não aceitáveis, levando em conta os requisitos estatutários, regulamentares, dos clientes, uso pretendido pelo consumidor e outros dados relevantes. Se um limite crítico for excedido ou violado, os produtos afetados são considerados como potencialmente inseguros e devem ser avaliados para liberação, 


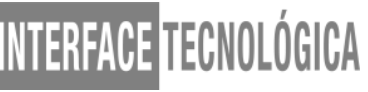

mediante o estabelecimento de procedimento para o tratamento destes produtos (ABNT NBR ISO 22000:2006).

Condições como temperatura, tempo, pressão, umidade, $\mathrm{pH}$, atividade de água, textura, aroma, viscosidade, acidez, cloro residual livre possibilitam que os limites sejam aplicáveis de acordo com a especificidade de cada operação (BRASIL, 1998). Os limites baseados em dados subjetivos, como inspeção visual, devem ser apoiados por instruções, especificações e treinamentos devidamente documentados.

\subsection{Quarto Princípio: Estabelecimento de Procedimentos de Monitoramento}

O monitoramento dos pontos críticos de controle deve ser estabelecido, para demonstrar que o ponto está sob controle, indicando se os limites críticos foram excedidos em tempo hábil de o produto ser isolado antes de ser usado ou consumido (ABNT NBR ISO 22000:2006).

As principais formas de monitoramento são: observação contínua, avaliação sensorial, análises laboratoriais e utilização de instrumentos de medida. É necessário o estabelecimento de um procedimento constando o que será monitorado, a sequência para o monitoramento e plano de amostragem, os limites críticos e o responsável treinado e apto no domínio da aplicação de técnicas e métodos.

\subsection{Quinto Princípio: Estabelecimento de ações corretivas}

Ação para eliminar a causa de uma não conformidade detectada ou outra situação indesejável é considerada uma ação corretiva. Ao ser constatado um desvio nos limites críticos estabelecidos, ações corretivas deverão ser tomadas imediatamente para a solução da situação.

As ações corretivas para eliminação da causa da não conformidade incluem análise de reclamação do consumidor, análise do resultado do monitoramento onde haja a possibilidade da perda do controle, a garantia da não recorrência da não conformidade e a eficácia das ações tomadas (ABNT NBR ISO 22000:2006).

Podem ser estabelecidas uma ou mais ações corretivas para a eliminação do perigo em um PCC, como nova calibração de equipamentos, rejeito de matéria prima e reprocesso, de 


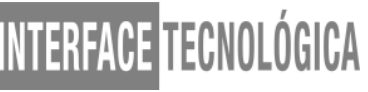

modo que o operador de processo tenha autorização para executar as ações. As ações devem assegurar que a causa da não conformidade seja identificada, que os parâmetros controlados no PCC sejam retornados ao controle e que recorrência seja prevenida (BRASIL, 1998).

\subsection{Sexto Princípio: Estabelecimento de Procedimentos de verificação}

Os procedimentos para as atividades de verificação servem para confirmar se os perigos à segurança de alimentos nos PCCs estão controlados, dentro dos níveis aceitáveis, as medidas de controle, limites críticos, monitoramento, ações corretivas, responsáveis e registros estão sendo eficazes dentro do plano APPCC.

Os resultados da verificação são registrados e comunicados à equipe de segurança de alimentos, permitindo análise dos dados em conformidade com o planejado e caso seja necessário são adotadas ações para alcançar a conformidade requerida (ABNT NBR ISO 22000:2006).

Fatores como pré-requisitos, normas, revisão dos limites críticos, aferição e calibração de equipamentos e instrumentos, análises sensoriais, físico, químicas e microbiológicas são observadas para determinar se os princípios do plano APPCC estão sendo cumpridos (BRASIL, 1998). A verificação permite avaliar a efetividade das ações corretivas tomadas e do funcionamento do APPCC.

\subsection{Sétimo Princípio: Registros de resultados}

Todos os dados, informações, resultados, desvios, causa dos desvios, ações corretivas, devem ser registrados em formulários, gráficos ou tabelas e mantidos em arquivo por no mínimo dois anos após o vencimento do produto. Documentos pertinentes à inspeção de matéria prima, tempo, temperatura, produto, pesagem, controle da cloração da água de abastecimento e treinamento de pessoal devem estar ordenados e de fácil acesso às consultas e vinculados com o plano APPCC (BRASIL, 1998).

O correto preenchimento e a manutenção dos registros são de grande importância para a comprovação de funcionamento do plano APPCC, além de servir de base para revisões futuras (BARRETO, J. et al., 2013). 


\section{CONCLUSÃO}

Os alimentos podem sofrer contaminações em todas as etapas do processo, por isso devem ser verificadas as condições de recebimento da matéria prima, os equipamentos utilizados na fabricação, a estrutura, o local do processamento e armazenagem, a higiene dos colaboradores, o material de embalagem a ser utilizado para que o produto possa ser fabricado com qualidade e segurança para o consumo humano.

O controle do processo de fabricação de alimentos influencia diretamente no produto final, pois para a obtenção da qualidade e excelência, todo o processo deve ser analisado, controlado e monitorado para que atenda à especificação técnica e aos requisitos desejados. $\mathrm{O}$ processo industrial funciona como uma engrenagem, onde a qualidade se faz desde a qualificação dos fornecedores de matéria prima, inspeção de recebimento, armazenamento, aprovação dos materiais e insumos, no cumprimento de procedimentos e roteiros de produção, na higienização adequada de equipamentos e instalações, no controle de processo rigoroso com colaboradores capacitados para a execução de suas atividades.

A qualidade se estende ainda no processo de embalagem, análise do produto final, estocagem, identificação e transporte adequado. Em todas as operações é fundamental a adoção de hábitos higiênicos para a prevenção de contaminações físicas, químicas ou microbiológicas.

O estudo com a metodologia sistemática do APPCC nos proporciona distinguir os pontos de controle dos pontos críticos de controle. Os pontos críticos de controle devem ser analisados e tomados às medidas corretivas necessárias para a contenção dos perigos, enquanto os pontos críticos de controle além de controlados devem ser monitorados rigorosamente, pois são pontos fundamentais de possíveis contaminações com consequências severas para a saúde humana.

Ao detectar uma falha no controle de algum ponto crítico de controle, o lote do produto deve ser imediatamente segregado para avaliação e considerado como produto potencialmente inseguro, até que seja realizada uma análise criteriosa para o destino final do produto.

A implantação do APPCC na indústria alimentícia propicia uma série de benefícios como aumento da qualidade do produto, maior produtividade com menor índice de retrabalho e redução do número de reclamações de consumidores. A certificação de uma empresa é um diferencial de qualidade que influencia no número de vendas, aceitabilidade e aprovação do 


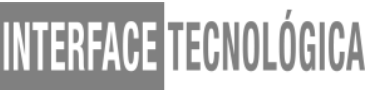

produto. O compromisso com a qualidade deve estar presente em todo o desenvolvimento do processo de fabricação para assegurar a confiabilidade dos clientes e fortalecer a imagem da empresa no mercado.

\section{REFERÊNCIAS}

ASSOCIAÇÃO BRASILEIRA DE NORMAS TÉCNICAS. NORMA BRASILEIRA ABNT NBR ISO 22000:2006. Sistemas de gestão da segurança de alimentos. Requisitos para qualquer organização na cadeia produtiva de alimentos. Disponível em:

$<$ https://pt.scribd.com/doc/57384728/NBR-ISO-22000-2006-Sistema-de-gestao-daseguranca-de-alimentos>. Acesso em: 17 de set. 2018.

BRASIL. Ministério da Saúde. Resolução de Diretoria Colegiada - RDC No 275, de 21 de outubro de 2002. Dispõe sobre o Regulamento Técnico de Procedimentos Operacionais Padronizados aplicados aos Estabelecimentos Produtores/Industrializadores de Alimentos e a Lista de Verificação das Boas Práticas de Fabricação em Estabelecimentos Produtores/Industrializadores de Alimentos. Diário Oficial da União. Brasília, DF. 23 de outubro de 2002. Seção 1, p. 126. Disponível em: <http://portal.anvisa.gov.br>. Acesso em: 17 de set. 2018.

Ministério da Agricultura, Pecuária e Abastecimento. Portaria n 46, de 10 de fevereiro de 1998. Sistema de Análise de Perigos e Pontos Críticos de Controle - APPCC a ser implantado, gradativamente, nas indústrias de produtos de origem animal sob o regime do Serviço de Inspeção Federal - SIF, de acordo com o Manual Genérico de Procedimentos.

Diário Oficial da União. Brasília, DF. 16 de março de 1998. Seção 1, p. 24. Disponível em: $<$ http://sistemasweb.agricultura.gov.br/sislegis/action/detalhaAto.do?method=consultarLegisl acaoFederal>. Acesso em: 02 de abr. 2018.

Ministério da Saúde. Portaria no 326, de 30 de julho de 1997. Regulamento Técnico; "Condições Higiênico-Sanitárias e de Boas Práticas de Fabricação para Estabelecimentos Produtores/Industrializadores de Alimentos". Diário Oficial da União. Brasília, DF. 01 de agosto de 1997. Disponível em: <http://bvsms.saude.gov.br>. Acesso em: 17 de set.2018.

Ministério da Saúde. Portaria no 1428, de 26 de novembro de 1993. Aprova, na forma dos textos anexos, o "Regulamento Técnico para Inspeção Sanitária de Alimentos", as "Diretrizes para o Estabelecimento de Boas Práticas de Produção e de Prestação de Serviços na Área de Alimentos" e o "Regulamento Técnico para o Estabelecimento de Padrão de Identidade e Qualidade (PIQ's) para Serviços e Produtos na Área de Alimentos". Determina que os estabelecimentos relacionados à área de alimentos adotem, sob responsabilidade técnica, as suas próprias Boas Práticas de Produção e/ou Prestação de Serviços, seus Programas de Qualidade, e atendam aos PIQ।'s para Produtos e Serviços na Área de Alimentos. Diário Oficial da União. Brasília, DF. 02 de dezembro de 1993. Disponível em: <http://portal.anvisa.gov.br>. Acesso em: 17 de set. 2018. 
Ministério da Agricultura, Pecuária e Abastecimento. Portaria no 368, de 4 de setembro de 1997. Aprova o Regulamento Técnico sobre as condições Higiênico-Sanitárias e de Boas Práticas de Fabricação para Estabelecimentos Elaboradores/Industrializadores de Alimentos. Diário Oficial da União. Brasília, DF. 08 de setembro de 1997. Disponível em: < https://www.diariodasleis.com.br>. Acesso em: 17 de set. 2018.

BARRETO, J. et al. Implantação da Análise de Perigos e Pontos Críticos de Controle (APPCC), Garantia da qualidade e segurança na indústria de alimentos. ACTA BIOMEDICA BRASILIENSIA. v.4, n.2, p. 72-80, 2013. Disponível em: < http://www.actabiomedica.com.br>. Acesso em: 25 de nov. 2018.

COLLETO, D. Gerenciamento da Segurança de Alimentos e da Qualidade na Indústria de Alimentos. Monografia apresentada ao curso de Engenharia de Alimentos, na Universidade Federal do rio Grande do Sul UFRGS. Porto Alegre - RS. 2012. 46. Disponível em: < https://www.lume.ufrgs.br>. Acesso em: 20 de set. 2018.

FIGUEIREDO, V. F.; COSTA N. Implantação do HAPPCC na indústria de alimentos. GESTÃO \& PRODUÇÃO v.8, n.1, p.100-111, 2001. Disponível em: <http://www.scielo.br>. Acesso em: 20 de set. 2018.

FURTINI, L. L. R.; ABREU, L.R. Utilização de APPCC na indústria de alimentos. CIÊNCIA E TECNOLOGIA. v.30, n.2, p. 358-363, 2006. Disponível em: < http://www.scielo.br>. Acesso em: 20 de set. 2018.

VERGARA, C. M. A. C. Gestão da qualidade na área de alimentos. NUTRIVISA. v.2, n.3, p. 99-100, 2015, 2016. Disponível em: < https://www.revistanutrivisa.com.br>. Acesso em: 25 de nov. 2018. 